Europhysics Letters

PREPRINT

\title{
Independence of the relaxation of a supercooled fluid of its microscopic dynamics: need for yet another extension of the mode-coupling theory
}

\author{
Grzegorz Szamel and Elijah Flenner \\ Department of Chemistry, Colorado State University, Fort Collins, CO 80525
}

PACS. 61.20. Lc -

PACS. 64.70. Pf -.

PACS. 61.43. Fs -

\begin{abstract}
Using Brownian Dynamics computer simulations we show that the relaxation of a supercooled Brownian system is qualitatively the same as that of a Newtonian system. In particular, near the so-called mode-coupling transition temperature, dynamic properties of the Brownian system exhibit the same deviations from power-law behavior as those of the Newtonian one. Thus, similar dynamical events cut off the idealized mode-coupling transition in Brownian and Newtonian systems. We discuss implications of this finding for extended modecoupling theory. In addition, we point out and discuss the difference between our findings and experimental results, and present an alternative interpretation of some of our simulation data.
\end{abstract}

Our present understanding of the glass transition owes a great deal to a series of computer simulation studies performed by Hansen and collaborators [1]. The main conclusion from these studies is that, at least for certain classes of materials, the glass transition is a two step process. The first step is the so-called kinetic glass transition occurring at a temperature $T_{c}$ above the laboratory glass transition temperature $T_{g}$. The kinetic transition is associated with a sudden freezing out of the usual hydrodynamic-like motions $[2,3]$. This transition is usually identified with an ergodicity breaking transition predicted by idealized mode-coupling theory (MCT) [4-6]. Below $T_{c}$ the dynamics is thought to be dominated by activated processes that are sometimes referred to as hopping events. It is claimed that extended MCT [7-9] is able to describe these events.

The kinetic transition has been carefully examined in computer simulation studies of Andersen, Kob, and coworkers [10-13]. The commonly accepted conclusion is that the idealized MCT provides a good description of this transition. In particular: the relaxation time shows a power-law temperature dependence with an exponent that is close to that predicted by the theory, the time dependence of the incoherent intermediate scattering function is reasonably well described by the so-called $\beta$-correlator of the theory, and the (effective) non-ergodicity parameter extracted from simulations agrees well with that calculated from the theory.

The above mentioned conclusion ignores a few caveats [14]. One of the more fundamental ones concerns an often overlooked confusion about the independence of the supercooled fluid's relaxation on its microscopic dynamics and MCT's description of this independence.

(c) EDP Sciences 
On one hand, it is commonly expected that standard Newtonian dynamics (ND) leads to the same glass transition scenario as Brownian dynamics (BD). Indeed, the first simulational indication of the independence of the kinetic glass transition on microscopic dynamics dates back to Hansen's investigations [3]. At about the same time it was shown theoretically that the idealized MCT leads to the same glass transition scenario for both Newtonian and Brownian dynamics [15]. More recently, Gleim et al. [16] performed a careful comparison of simulation results obtained using ND and so-called stochastic dynamics (SD) [17]. Again, they showed that the kinetic glass transition scenario is remarkably independent of the microscopic dynamics. These simulational and theoretical indications of the independence of the kinetic glass transition scenario of the fluid's microscopic dynamics justified using the MCT to analyze the colloidal glass transition. The result of this analysis was very favorable for the theory [19].

It has been remarked [19] that in real colloidal systems hopping events are suppressed and thus the experimental colloidal glass transition coincides with the idealized MCT ergodicity breaking transition. This is good news for the theory for the following reason: MCT for Brownian systems cannot be extended in the same way as MCT for Newtonian ones. In the latter case the extension and the resulting cut-off of the ergodicity breaking transition is achieved through coupling to current modes that are defined in terms of particle's velocities. Since in Brownian systems there are no velocities (particle's positions are the only dynamic variables), there are no currents to add to the standard set of slow variables, and the usual way to extend MCT fails. However, the apparent absence of hopping events in experimental colloidal systems made this potential problem irrelevant. Indeed, it has been sometimes claimed that the colloidal glass transition is completely described by the idealized MCT.

In contrast to this seemingly consistent picture, it was already clear from Hansen's early studies that Brownian systems commonly investigated in computer simulations [20] do not undergo the ergodicity breaking transition. In particular, Löwen et al. [3] saw very similar hopping events for both Newtonian and Brownian dynamics. It has to be admitted that these events were seen at the lowest temperature, and the systems studied were probably on the verge of being equilibrated. However, similar hopping events were seen in a later study [18]. In addition, it is clear from Gleim et al. [16] that similar deviations from idealized modecoupling-like behavior are present in both Newtonian and stochastic systems.

It should be emphasized that Gleim's study, although indicative, does not really address the most important, fundamental point: qualitative disagreement between simulations (that show absence of the ergodicity breaking transition) and MCT for Brownian systems (that, at present, cannot be extended). This is due to the fact that MCT for stochastic systems could, in principle, be extended in the same way as one for Newtonian systems (in both cases one can define current modes using particle's velocities). It is this fact that motivated us to perform a large scale Brownian dynamics study of a model used before in Newtonian and stochastic simulations: the Kob-Andersen [10] binary Lennard-Jones mixture [21].

We consider an 80:20 mixture of 1000 Lennard-Jones particles. The interaction between the particles is given by $V_{\alpha \beta}=4 \epsilon_{\alpha \beta}\left[\left(\sigma_{\alpha \beta} / r\right)^{12}-\left(\sigma_{\alpha \beta} / r\right)^{6}\right]$ where $\alpha, \beta \in\{A, B\}$ and $\epsilon_{A A}=1.0$, $\sigma_{A A}=1.0, \epsilon_{A B}=1.5, \sigma_{A B}=0.8, \epsilon_{B B}=0.5$, and $\sigma_{B B}=0.88$. All the interaction potentials are cut-off at $2.5 \sigma_{\alpha \beta}$. The box length of the (cubic) simulation box is equal to $9.4 \sigma_{A A}$.

The Lennard-Jones binary mixture considered here was extensively investigated using Newtonian $[10,11,13]$ and stochastic [16] dynamics. The main finding is that as temperature approaches $T_{c}=0.435 \epsilon_{A A} / k_{B}$ self-diffusion coefficients and relaxation times change rapidly; their temperature dependence can be well fitted by power laws, in agreement with the MCT. However, in contrast to the theoretical prediction, the exponents for self-diffusion coefficients and relaxation times are different. Moreover, the ergodicity breaking transition is avoided, 
instead there appears to be a crossover to a different relaxation scenario [1]. The temperature obtained from power-law fits is commonly referred to as the mode-coupling transition temperature $T_{c}$. It should be emphasized that it is quite different from the temperature that the MCT would predict using realistic structure factors as input [11]. Near $T_{c}$, time-dependence of the correlators is well described by the MCT [12]; also wave-vector dependence of the effective non-ergodicity parameter is well described by the theory. All of these conclusions are valid for both Newtonian and stochastic dynamics.

Here we consider BD: the particles are performing interacting diffusive motion; they move under the influence of deterministic and stochastic forces. Explicitly, for the $i$ th particle,

$$
\dot{\vec{r}}_{i}=-\frac{1}{\xi_{0}} \nabla_{i} \sum_{j \neq i} V_{\alpha \beta}\left(\left|\vec{r}_{i}-\vec{r}_{j}\right|\right)+\vec{\eta}_{i}(t)
$$

where $\xi_{0}=1.0$ is the friction coefficient of an isolated particle and random noise $\eta_{i}$ satisfies the fluctuation-dissipation theorem, i.e.

$$
\left\langle\vec{\eta}_{i}(t) \vec{\eta}_{j}\left(t^{\prime}\right)\right\rangle=2 D_{0} \delta\left(t-t^{\prime}\right) \delta_{i j} I
$$

In Eq. (2) $D_{0}=k_{B} T / \xi_{0}$ is the diffusion coefficient of an isolated particle and $I$ is a unit tensor. One should note that the equations of motion allow diffusive motion of the system's center-of-mass. All results presented here pertain to the motion relative to the center-of-mass.

In the following we use reduced units with $\sigma_{A A}, \epsilon_{A A}, \epsilon_{A A} / k_{B}$, and $\sigma_{A A}^{2} \xi_{0} / \epsilon_{A A}$ being the units of length, energy, temperature, and time, respectively.

Equations of motion (11) were solved using a Heun algorithm [22]. We used a very small time step of $5 \times 10^{-5}$. To check that the equilibrium and dynamic properties are independent of the time step, we run a couple of production runs at twice smaller time step. We simulated the system at temperatures $1.0,0.8,0.6,0.55,0.5,0.47,0.45$ and 0.44 . At each temperature we performed a long equilibration run and several production runs (equilibration runs were at least as long as production runs). The results presented were obtained by averaging over different production runs.

One should note that at the time of the Gleim et al. [16] investigation the present study was not feasible. The improvement in computer speed allowed us to perform very long Brownian dynamics runs (up to $6 \times 10^{8}$ steps).

We present here data for the mean squared displacement (MSD), self-diffusion coefficient, intermediate scattering function, $\alpha$ relaxation time, non-Gaussian parameter, and the self part of the van Hove correlation function. The data for the the first four quantities are similar to the previous results for Newtonian and stochastic dynamics. For the non-Gaussian parameter we show for the first time that its amplitude increases according to a power law, also the characteristic time scale seems to increase according to a power law. The self part of the van Hove function shows clearly that a qualitatively different relaxation scenario sets in near $T_{c}$.

In Fig. 17 we show the MSD for the A particles. At the shortest times the MSD grows linearly with time: the motion is diffusive with diffusion coefficient equal to $D_{0}=k_{B} T / \xi_{0}$. In the reduced units used here $D_{0}$ decreases by a factor of approximately 2 over the range of temperatures studied. At longer times the MSD changes very slowly due to transient "cage" localization. We obtain the self-diffusion coefficients from the diffusive behavior of the MSD seen at the longest times. In Fig. 10 we show the temperature dependence of the self-diffusion coefficients for both A and B particles: it can be fitted by power laws up to $\left(T-T_{c}\right) / T_{c} \approx 0.08$ (unless stated otherwise, all fits were performed using temperature range $0.47 \leq T \leq 0.8$ ), and the exponents are essentially the same as in ND. Closer to $T_{c}$, the self-diffusion coefficients deviate from power laws in a way similar to that found in Newtonian systems. 

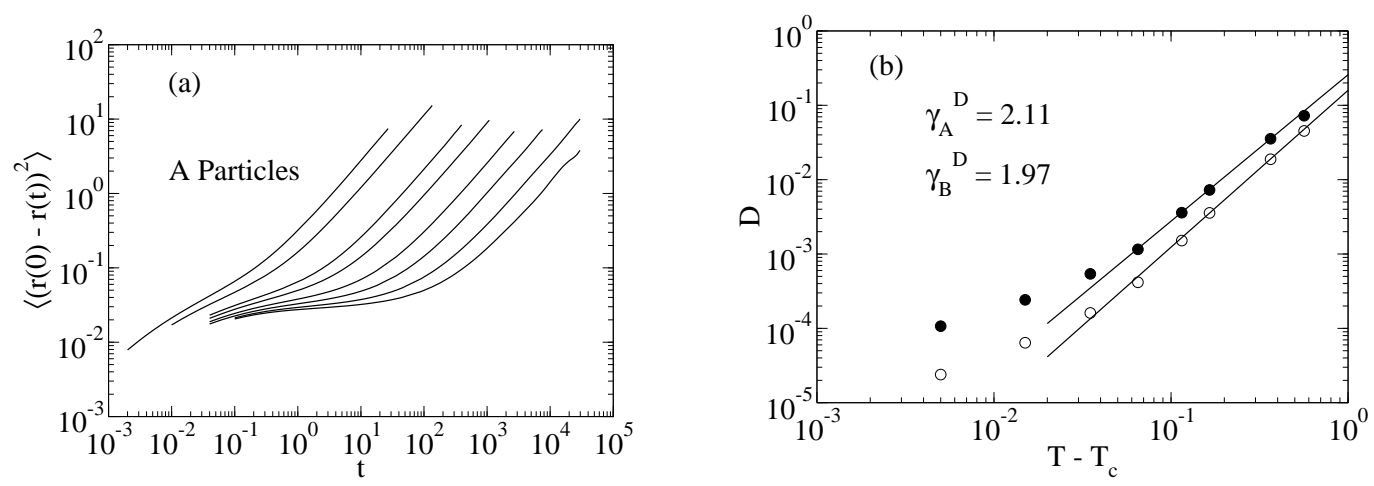

Fig. 1 - (a) Time dependence of the mean squared displacement; $T=1,0.8,0.6,0.55,0.5,0.47,0.45$, 0.44. (b) Temperature dependence of the self-diffusion coefficients; open symbols: A particles, closed symbols: B particles; lines: power-law fits, $D \propto\left(T-T_{c}\right)^{\gamma^{D}}$; exponents $\gamma^{D}$ are listed in the figure.

In Fig. 2a we show time dependence of the incoherent intermediate scattering function, $F_{s}(k ; t)$, for all temperatures for the $A$ particles at a wavevector close to the peak in the $A A$ partial structure factor. Qualitatively, we see that our results resemble those obtained for stochastic dynamics [16]: on approaching the mode coupling transition temperature a plateau develops while the characteristic relaxation time sharply increases. We follow earlier workers and define the $\alpha$ relaxation time as the time at which $F_{s}$ decays to $e^{-1}$. The temperature dependence of the relaxation times for $A$ and $B$ particles is shown in Fig. 2b. Again, the temperature dependence can be fitted by power laws, and the exponents are essentially the same as in Newtonian systems. One should note that, as in Newtonian and stochastic systems, we see a violation of the MCT's $\alpha$-scale universality: power laws for self-diffusion coefficients are different from those for relaxation times. As for self-diffusion coefficients, close to $T_{c}$ relaxation times clearly deviate from power laws.

In Fig. 3a we show the non-Gaussian parameter $\alpha_{2}(t)=3\left\langle\Delta r^{4}(t)\right\rangle / 5\left\langle\Delta r^{2}(t)\right\rangle^{2}-1$. As found earlier [10,23], $\alpha_{2}$ is zero at short times, has a maximum at intermediate times and decays to zero on time scale somewhat shorter than the $\alpha$ relaxation time scale. Note that
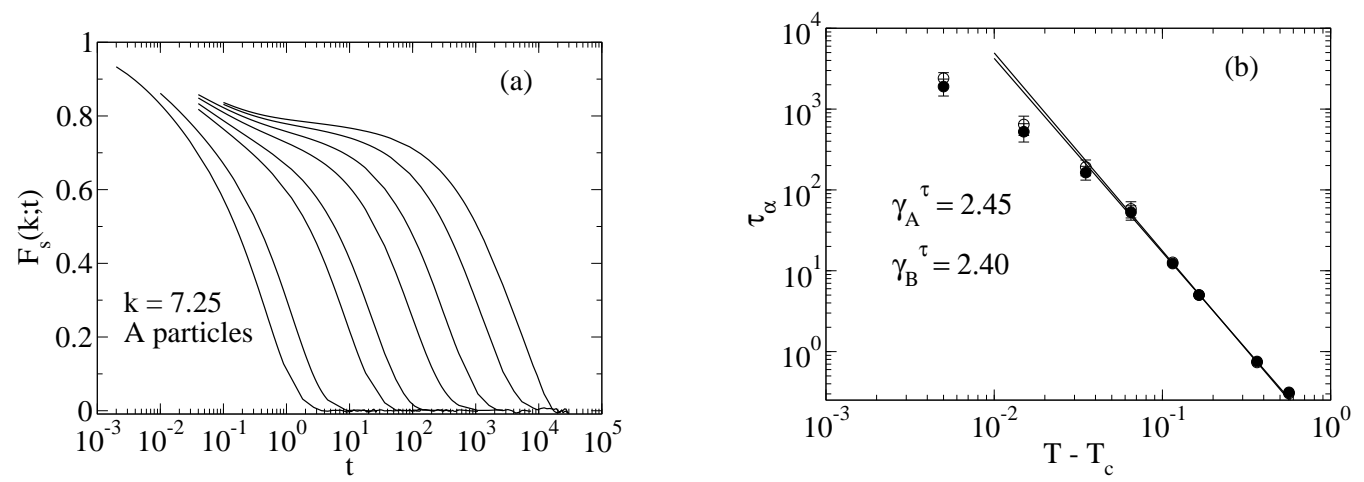

Fig. 2 - (a) Time dependence of the incoherent intermediate scattering function; $T=1,0.8,0.6$, $0.55,0.5,0.47,0.45,0.44$. (b) Temperature dependence of the $\alpha$ relaxation times; open symbols: A particles, closed symbols: B particles; lines: power-law fits, $\tau_{\alpha} \propto\left(T-T_{c}\right)^{-\gamma^{\tau}}$. 

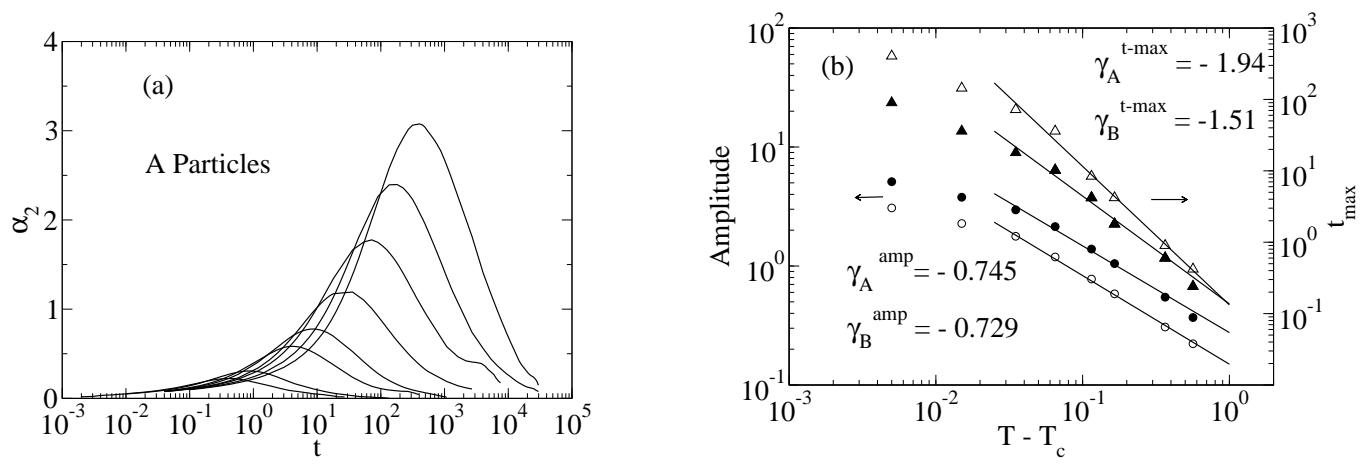

Fig. 3 - (a) Time dependence of the non-Gaussian parameter function; $T=1,0.8,0.6,0.55,0.5$, $0.47,0.45,0.44$. (b) Temperature dependence of the amplitude of the non-Gaussian parameter and its characteristic time; open symbols: A particles, closed symbols: B particles.

the maximum value of $\alpha_{2}$ is somewhat larger than in Newtonian systems. In Fig. 3b we show the temperature dependence of the maximum value of $\alpha_{2}$ and the time at which it has the maximum value. Both quantities follow power laws in the same temperature range as selfdiffusion coefficients and relaxation times. In case of the amplitude this is different from the prediction of the mode-coupling theory [24]: the theory predicts that the amplitude of the non-Gaussian parameter saturates as temperature approaches $T_{c}$. In case of the characteristic time we again see a violation of the $\alpha$-relaxation universality: the temperature dependence of the characteristic time of the non-Gaussian parameter is similar to that of the self-diffusion coefficients and different from that of the scattering function relaxation time. Finally, both the amplitude and the characteristic time deviate from power law behavior close to $T_{c}$.

The last quantity presented here, shown in Fig. 目 is the self part of the van Hove correlation function, $G_{s}^{\alpha}(r, t)=\frac{1}{N_{\alpha}}\left\langle\sum_{i=1}^{N_{\alpha}} \delta\left(r-\left|\vec{r}_{i}(t)-\vec{r}_{i}(0)\right|\right)\right\rangle$, where $\alpha \in\{A, B\}$. We show $4 \pi r^{2} G_{s}^{A}(r, t)$ for times equal to $0.5 \tau_{\alpha}, \tau_{\alpha}, 2 \tau_{\alpha}$ and $3 \tau_{\alpha}$ at two different temperatures, $T=1$ (Fig. 4 4 ) and $T=0.44$ (Fig. 四). We clearly see two different relaxation scenarios. At the higher temperature the position of the peak of the van Hove function moves with time and
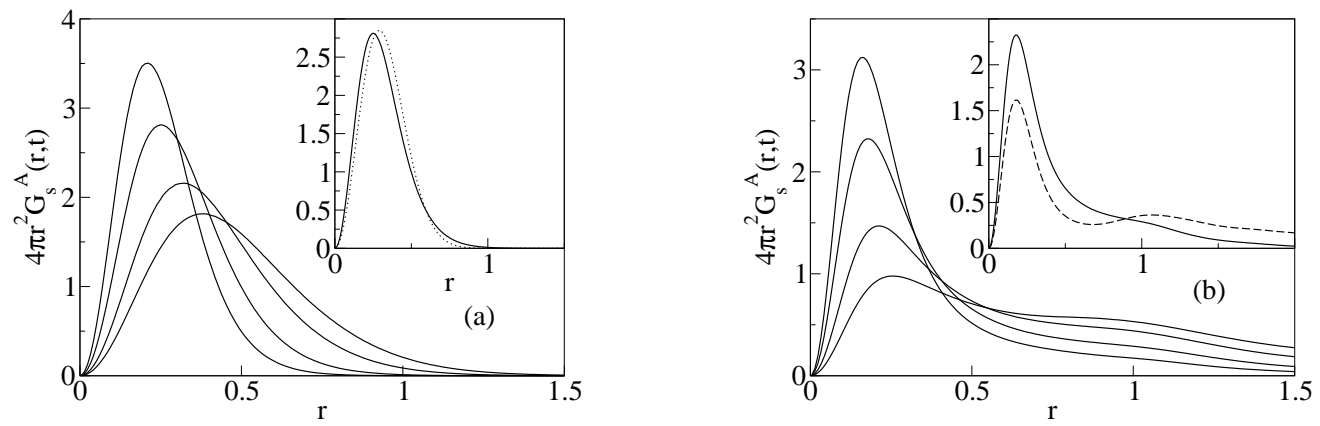

Fig. $4-$ (a) $4 \pi r^{2} G_{s}^{A}(r, t)$ at $T=1$ for $t=0.5 \tau_{\alpha}, \tau_{\alpha}, 2 \tau_{\alpha}, 3 \tau_{\alpha}$ (at $T=1 \tau_{\alpha}=0.304$ ); insert: $4 \pi r^{2} G_{s}^{A}(r, t)$ at $T=1$ for $t=\tau_{\alpha}$ (solid line) and Gaussian probability distribution with the same $\left\langle r^{2}\right\rangle$ (dotted line). (b) $4 \pi r^{2} G_{s}^{A}(r, t)$ at $T=0.44$ for $t=0.5 \tau_{\alpha}, \tau_{\alpha}, 2 \tau_{\alpha}, 3 \tau_{\alpha}$ (at $\left.T=0.44 \tau_{\alpha}=2409\right)$; insert: $4 \pi r^{2} G_{s}^{A}(r, t)$ (solid line) and $4 \pi r^{2} G_{s}^{B}(r, t)$ (dashed line) at $T=0.44$ for $t=\tau_{\alpha}$. 
the shape of the correlation function is close to Gaussian. At the lower temperature the position of the peak is almost time-independent. In addition, a pronounced shoulder develops at distances around 1 . In the same time range $4 \pi r^{2} G_{s}^{B}$ has a well developed peak at distances around 1 (see insert). Similar features in the van Hove function were seen earlier $[2,3]$ but only at the lowest temperature. In this study an arrested peak of the van Hove function, a shoulder in $4 \pi r^{2} G_{s}^{A}$, and a secondary peak in $4 \pi r^{2} G_{s}^{B}$ were seen both at $T=0.44$ and $T=0.45$ (not shown). Earlier studies [2,3] interpreted such features as evidence of an activated hopping process. Note that at $T=0.44$ for the longest time shown in Fig. $4 \mathrm{~b}, t=3 \tau_{\alpha}$, the van Hove function is very clearly far from Gaussian although the non-Gaussian parameter for $t=3 \tau_{\alpha}$ is well past its maximum. The non-Gaussian shape of the van Hove function suggests that pronounced dynamic heterogeneity is still present on this time scale.

In summary, we clearly showed that in Brownian systems, as found earlier in Newtonian and stochastic systems, the kinetic glass transition does not coincide with the ergodicity breaking. The power law dependence of transport properties, predicted by the mode-coupling theory, is valid over two to three decades of change of the self-diffusion coefficients and relaxation times. Close to $T_{c}$ there are departures from the power laws that cannot be (even qualitatively) explained by the mode-coupling theory. The deviations from power laws are similar to those found for Newtonian and stochastic systems [25]. This confirms an earlier observation [3] that similar dynamic events are responsible for relaxation in Newtonian and Brownian systems below the kinetic glass transition temperature.

The results presented here suggest a need for an extension of the idealized mode-coupling theory that is applicable to both Newtonian and Brownian systems. At present it is unclear how to proceed toward this goal. It should be noted in this context that a mode-coupling-like theory for kinetic Ising models with stochastic dynamics has been extended [26]. The final formulas of the extended theory resemble those of the extended mode-coupling theories [7-9] although they are derived in a completely different way.

We would like to point out the disagreement between our findings and the experimental results. The most striking difference is between our Figs. 10 and $2 \mathrm{~b}$ and Fig. 11 of van Megen et al. [27]: our results violate the $\alpha$-scale universality and deviate from power laws close to the transition temperature whereas van Megen's results show the same power laws for the self-diffusion coefficients and the relaxation time and do not show any deviation from power-law behavior [28]. There could be two possible sources of this disagreement. First, our particles interact via Lennard-Jones potentials whereas van Megen's colloidal particles have hard-sphere-like interactions. Second, we did not include hydrodynamic interactions that are known to be very important in real hard-sphere suspensions. Instead of speculating on the importance of these two issues we would like to point out that neglecting hydrodynamic interactions it should be possible to simulate a polydisperse hard sphere system that quantitatively models the experimental system of Ref. [27]. Such a simulation could exclude the difference in the interaction potentials as the source of the qualitative disagreement between simulations and experiment.

Finally, we would like to note that an alternative interpretation [29] of some of our simulation data is possible: if the transition temperature is a fit parameter, reasonable power-law fits can be obtained for self-diffusion coefficients and relaxation times in the low temperature region, $0.44 \leq T \leq 0.6$. These fits result in a considerably lower transition temperature $T_{c}^{\prime} \approx 0.414$ ( $T_{c}^{\prime}$ depends slightly on the quantity fitted) and the exponents $\gamma_{A}^{D}=2.56$, $\gamma_{B}^{D}=2.09, \gamma_{A}^{\tau}=3.04$, and $\gamma_{B}^{\tau}=2.93$. While these fits suggest that the power-law temperature dependence of the self-diffusion coefficients and relaxation times may extend to temperatures lower than reported previously $[10,16]$, accepting this alternative interpretation would open a new set of questions. Here we pose only two of them: First, does the kinetic transition 
temperature depend on the microscopic dynamics in stark disagreement with the prediction of the idealized MCT [15], or do the previously reported data for ND [10] and SD [16] have to be re-interpreted as well? Second, how to reconcile the validity of the idealized MCT-like power-law temperature dependence and the strikingly non-Gaussian van Hove function that suggests presence of hopping-like processes that are absent in the idealized MCT?

\section{ACKNOWLEDGMENT}

Support by NSF Grant No. CHE 0111152 is gratefully acknowledged.

\section{REFERENCES}

[1] J.P. Hansen, Physica A 201, 138 (1995).

[2] J.N. Roux, J.L. Barrat, and J.P. Hansen, J. Phys. Cond. Matt. 1, 7171 (1989); J.L. Barrat, J.N. Roux, and J.P. Hansen, Chem. Phys. 149, 197 (1990).

[3] H. Löwen, J.P. Hansen, and J.N. Roux, Phys. Rev. A 44, 1169 (1991).

[4] U. Bengzelius, W. Götze, and A. Sjölander, J. Phys. C 17, 5915 (1984).

[5] S.P. Das, G.F. Mazenko, S. Ramaswamy, and J.J. Toner, Phys. Rev. Lett. 54, 118 (1985).

[6] To distinguish between the original MCT [4,5] and its later, extended versions [7-9], whenever ambiguity can arise, we will refer to the former one as "the idealized mode-coupling theory".

[7] S.P. Das and G.F. Mazenko, Phys. Rev. A 34, 2265 (1986).

[8] W. Götze and L. Sjögren, Z. Phys. B 65, 415 (1987).

[9] R. Schmitz, J. W. Dufty, and P. De, Phys. Rev. Lett. 71, 2066 (1993).

[10] W. Kob and H.C. Andersen, Phys. Rev. E 51, 4626 (1995); Phys. Rev. E 52, 4134 (1995).

[11] M. Nauroth and W. Kob, Phys. Rev. E 55, 657 (1997).

[12] W. Kob, M. Nauroth, and F. Sciortino, J. Non-Cryst. Solids 307-310, 181 (2002).

[13] For a review see, e.g., W. Kob, J. Phys. Cond. Matter 11, R85 (1999).

[14] The most often mentioned one concerns the location of the kinetic transition: when computer simulated structure factors (that constitute the only input required by the theory) are used in mode-coupling calculations the resulting transition temperature for the Lennard-Jones system studied by Kob et al. is overestimated by a factor of two [11].

[15] G. Szamel and H. Löwen, Phys. Rev. A 44, 8215 (1991).

[16] T. Gleim, W. Kob, and K. Binder, Phys. Rev. Lett. 81, 4404 (1998).

[17] Stochastic dynamics is a Newtonian dynamics with addition of a Gaussian distributed white noise force and a damping force proportional to the particle's velocity.

[18] S. Sanyal and A.K. Sood, Phys. Rev. E 57, 908 (1998).

[19] W. van Megen, Transport Theory and Stat. Phys. 24, 1017 (1995).

[20] Note that in computer simulations of colloidal suspensions hydrodynamic interactions are usually neglected.

[21] We are using the Kob-Andersen mixture rather than a more realistic screened-Coulomb (i.e. Yukawa) system in order to be able to directly compare our Brownian Dynamics results to those obtained for the same system using Newtonian [10] and stochastic [16] dynamics.

[22] W. Paul and D.Y. Yoon, Phys. Rev. E 52, 657 (1995).

[23] W. Kob et al, Phys. Rev. Lett. 792827 (1997).

[24] M Fuchs, W. Götze, M.R. Mayr, Phys. Rev. E 58, 3384 (1998).

[25] T. Gleim, PhD Dissertation, unpublished.

[26] S.J. Pitts and H.C. Andersen, J. Chem. Phys. 114, 1101 (2001).

[27] W. van Megen, T.C. Mortensen, S.R. Williams and J. Müller, Phys. Rev. E 58, 6073 (1998).

[28] Note that simulational results vary over approximately three decades whereas the experimental ones vary over approximately four decades. However, if long-time diffusion coefficients are normalized by the short-time ones, in both cases the results vary over about three decades.

[29] We thank an anonymous referee for the suggestion. 